1 Universidade do Estado da Bahia (Uneb) - Salvador (BA), Brasil. Orcid: https://orcid. org/0000-0002-2561$088 \mathrm{X}$

thais.aranha@gmail.com

2 Universidade Federal da Bahia (UFBA), Instituto de Saúde Coletiva (ISC) Salvador (BA), Brasil. Orcid: https://orcid. org/0000-0002-14768649

schaves@ufba.br

3 Universidade Federal da Bahia (UFBA), Instituto de Saúde Coletiva (ISC) Salvador (BA), Brasil. Orcid: https://orcid. org/0000-0002-9285$194 X$

anamariafsl@gmail.com

4 Universidade do Estado da Bahia (Uneb) - Salvador (BA), Brasil.

Orcid: https://orcid org/0000-0001-86202313

carlamarialima_4@hotmail. com

5 Universidade Federal da Bahia (UFBA), Instituto de Saúde Coletiva (ISC) Salvador (BA), Brasil. Orcid: https://orcid. org/0000-0002-83362300

sissesan@hotmail.com

\section{O financiamento federal da política de saúde bucal no Brasil entre 2003 e 2017}

\author{
The federal funding of the oral health policy in Brazil between 2003 \\ and 2017
}

Thais Regis Aranha Rossi ${ }^{\mathbf{1}}$, Sonia Cristina Lima Chaves ${ }^{\mathbf{2}}$, Ana Maria Freire de Lima Almeida $\mathbf{3}$, Carla Maria Lima Santos ${ }^{4}$, Sisse Figueredo de Santana ${ }^{5}$

DOI: 10.1590/0103-1104201811903

RESUMO Este estudo descreveu e analisou os repasses financeiros federais para a Política Nacional de Saúde bucal (PNSB) no período 2003-2017. Realizou-se análise retrospectiva do financiamento federal da PNSB com dados coletados da base do Fundo Nacional de Saúde, nas rubricas relacionadas com a saúde bucal com correção de valores pelo Índice de Preços ao Consumidor (IPCA) até 2017. A série histórica dos repasses para a atenção em saúde bucal no período 2003-2017 mostrou um gasto federal crescente até 2012, com queda no início de 2013, manutenção de valores de 2013 a 2016 e discreta redução em 2017, chegando a $\mathrm{R} \$ 1.059 .555 .548,48$ neste último ano. Isso significou que o percentual de repasse do governo federal para a saúde bucal em relação ao repasse total passou de $0,62 \% \mathrm{em}$ 2003 para 1,63\% em 2017. Observou-se um aumento da participação percentual do financiamento da Atenção Especializada, principalmente a partir de 2013, passando de 2,3\% em 2008 para 21,6\% em 2017. A Atenção Básica recebia 97,3\% do financiamento federal em 2003 e apenas 78,3\% em 2017. Medidas de austeridade fiscal e mudanças nos blocos de financiamento podem indicar um caminho institucionalizado de redução dos repasses federais na saúde que poderão impactar nessa política setorial específica.

PALAVRAS-CHAVE Saúde bucal. Financiamento governamental. Financiamento da assistência à saúde. Serviços de saúde bucal. Política de Saúde.

ABSTRACT This study described and analyzed the federal financial transfers for the Oral Health Policy in Brazil (PNSB), in the period 2003-2017. A retrospective analysis of the federal funding of the PNSB was carried out with data collected from the National Health Fund base under the headings related to oral health with correction of values by Consumer Price Index (IPCA) until 2017. The historical series of spending for oral health care in 2003-2017 showed a growing federal spending up to 2012, falling at the beginning of 2013, maintaining values from 2013 to 2016 and a slight reduction in 2017, reaching $R \$$ $1,059,555,548.48$ this past year. This meant that the percentage of federal government transfer to oral health in relation to the total transfer rate increased from $0.62 \%$ in 2003 to $1.63 \%$ in 2017 . There was an increase in the percentage share of Specialized Care financing, mainly as of 2013, from 2.3\% in 2008 to $21.6 \%$ in 2017. Primary Care received $97.3 \%$ of federal funding in 2003 and only $78.3 \%$ in 2017 . The investment has been low. Fiscal austerity measures and changes in funding blocks may indicate an institutionalized path of reduction of federal transfer in health, which may impact this particular sectoral policy.

KEYWORDS Oral health. Financing, government. Healthcare financing. Dental health services. Health policy. 


\section{Introdução}

O modelo de financiamento da saúde adotado no Brasil é historicamente marcado pelo aporte de recursos públicos. A presença de financiamento privado sempre contou com fortes subsídios e subvenções diretas e indiretas do Estado. A adoção de repasses financeiros aplicados de forma transparente, capaz de atender à aplicação racional dos recursos na rede de atenção à saúde, potencializa os princípios doutrinários e organizativos do Sistema Único de Saúde (SUS), além do controle social que possibilita à sociedade civil o acompanhamento do fluxo orçamentário na organização da oferta de serviços de saúde'.

A Constituição Federal brasileira de 1988 demarcou o orçamento da saúde na seguridade social com recursos provenientes da União, estados, Distrito Federal (DF), municípios e contribuições sociais². As Leis Orgânicas da Saúde (8.080/1990 e 8.142/1990), a Lei Orgânica da Seguridade Social (8.212/1991), a Lei sobre os Benefícios da Previdência Social (8.123/1991), a Lei Orgânica da Assistência Social (8.742/1993), o Decreto $\mathrm{n}^{0}$ 1.232/1994 com as Normas de repasses do Fundo Nacional de Saúde (FNS), a Emenda Constitucional no 29/2000, o Decreto $n^{0} 7.508 / 2011$, a Lei ${ }^{0} 141 / 2012$ e o Decreto $n^{0} 7.827 / 2012$ configuram, com a Constituição Federal de 1988, a legislação principal sobre a saúde no Brasil com os dispositivos legais para repasses financeiros ${ }^{3,4}$.

Com o processo de implementação do SUS e a consequente municipalização dos serviços, o financiamento federal, apesar de ainda representar a principal fonte de recursos, reduziu sua participação no financiamento da saúde nas últimas décadas, com recursos federais financiando, respectivamente, $60,7 \%, 63,8 \%, 53,7 \%$ e $58,3 \%$ do gasto público em saúde nos anos de 1994, 1995, 1996 e 2000, caracterizando um decréscimo no que se refere ao financiamento federal 5 .

O financiamento, como componente do sistema de saúde, expressa o grau de priorização de determinada ação do Estado. Analisar o financiamento do sistema de saúde implica compreender quais os compromissos políticos assumidos pelos gestores ${ }^{6}$, além de fornecer estudos econômicos que auxiliem policymakers nas tomadas de decisão mesmo em contexto de recursos financeiros limitados ${ }^{7}$. Nos países da América do Sul, chama a atenção a heterogeneidade dos gastos em saúde, bem como as diferenças entre gasto público e gasto privado que inclui o gasto das famílias via financiamento privado que é realizado mediante desembolsos diretos (out of pocket) ou por meio do pré-pagamento de planos e seguros privados de saúde. No caso do Brasil, apesar da existência de um sistema universal, o percentual do gasto público em saúde é inferior ao de países como a Colômbia, o Uruguai e a Argentina ${ }^{8}$.

Estudos sobre financiamento da saúde no âmbito federal no Brasil vêm sendo desenvolvidos e trazem diversas contribuições para a literatura científica, a exemplo dos trabalhos sobre o financiamento da Atenção Básica (AB) ${ }^{7-10}$, a utilização dos serviços por sistemas de financiamento no período de 1998 a $2008^{11}$; as características do Sistema de Informações sobre Orçamentos Públicos de Saúde (Siops) ${ }^{4}$, o mix público-privado no sistema de saúde brasileiro ${ }^{12}$, os impactos da vinculação de receitas e das transferências condicionadas à oferta do financiamento da saúde ${ }^{13}$.

Os estudos sobre financiamento da saúde bucal no SUS abordam os repasses no nível municipal - reforçando que o município continua sendo o principal provedor do custeio da saúde bucal na $\mathrm{AB}^{14}$-, o custeio de procedimentos específicos de ortodontia ${ }^{15}$, a evolução dos incentivos federais setoriais ${ }^{15}$, estudo retrospectivo dos procedimentos básicos, de Média e Alta Complexidade e ações estratégicas de 1997 a $2005^{17}$. Não foram encontrados estudos relativos a períodos mais recentes que analisassem o financiamento das políticas de saúde bucal quanto aos repasses federais.

Diante do exposto, este estudo tem como pergunta orientadora: como se dá o financiamento federal para a política de saúde bucal no Brasil? Este artigo descreveu e analisou os repasses financeiros da União para a Política Nacional de Saúde Bucal (PNSB) entre 2003 e 2017. 


\section{Metodologia}

Realizou-se estudo retrospectivo sobre o financiamento federal da PNSB, em que foram coletados dados da base do FNS quanto aos repasses realizados nas rubricas relacionadas com a saúde bucal no período de 2003 a 2017. Cabe destacar que este estudo faz parte do eixo temático 'Análise de Políticas de saúde bucal no Brasil' do Observatório de Análise Política em Saúde do Instituto de Saúde Coletiva da Universidade Federal da Bahia (UFBA).

Os recursos para a PNSB estão garantidos em três blocos: AB, Média e Alta Complexidade e gestão, e são administrados pelo FNS. O Conselho Nacional de Saúde (CNS) é responsável pela fiscalização; e o Ministério da Saúde, órgão gestor do FNS, monitora a aplicação ${ }^{3}$. No FNS, os repasses financeiros estavam designados por blocos de financiamento, conforme quadro 1. Em relação ao custeio, o bloco de financiamento federal da $\mathrm{AB}$ para estados, DF e municípios é subdividido em: a) Equipes de Saúde Bucal (ESB) Unidade Odontológica Móvel (UOM); b) Incentivo adicional de saúde bucal; e c) Saúde bucal. O custeio para prestadores federais conveniados inclui: a) Incentivo adicional UOM e b) Saúde bucal.

Quadro 1. Forma de repasses fundo a fundo de financiamento de custeio e investimento por rubrica específica no período 2003-2017, para a saúde bucal na Atenção Básica e Especializada

\begin{tabular}{|c|c|}
\hline & Repasses fundo a fundo (custeio e investimento) \\
\hline \multirow{7}{*}{$\begin{array}{l}\text { 1) Bloco de Financiamento da } \\
\text { Atenção Básica }\end{array}$} & 1.1) Repasses a estados, DF e municípios \\
\hline & a) Equipe de Saúde Bucal - Unidade Odontológica Móvel (UOM) \\
\hline & b) Incentivo Adicional de Saúde Bucal \\
\hline & c) Saúde Bucal \\
\hline & 1.2.) Prestadores Federais Conveniados \\
\hline & a) Incentivo Adicional UOM \\
\hline & b) Saúde Bucal \\
\hline \multirow{13}{*}{$\begin{array}{l}\text { 2) Bloco de Financiamento - Mé- } \\
\text { dia e Alta Complexidade Ambu- } \\
\text { latorial e Hospitalar }\end{array}$} & 2.1) Repasses a estados, DF e municípios \\
\hline & a) CEO - Centro de Especialidades Odontológicas - municipal \\
\hline & b) CEO estadual \\
\hline & $\begin{array}{l}\text { c) Fundos de Ações Estratégicas e Compensações - Faec AlH (Autorização de } \\
\text { Internação Hospitalar) - Cirurgia Orofacial }\end{array}$ \\
\hline & d) Faec AlH - Tratamentos odontológicos \\
\hline & e) Faec SIA - OPM em Odontologia \\
\hline & f) Faec SIA - Tratamentos odontológicos \\
\hline & 2.2) Prestadores Federais Conveniados \\
\hline & a) CEO municipal \\
\hline & b) CEO estadual \\
\hline & 3) Faec AlH - Cirurgia Orofacial - Prestadores Federais Conveniados \\
\hline & a) Faec AlH - Tratamentos odontológicos - Prestadores Federais Conveniados \\
\hline & b) Faec SIA - Tratamentos odontológicos - Prestadores Federais Conveniados \\
\hline
\end{tabular}




\begin{tabular}{l} 
Quadro 1. (cont.) \\
$\begin{array}{ll}\text { II. Investimento (capital) } & \text { 4) Atenção Básica em Saúde Bucal (Aquisição de equipamentos e material perma- } \\
\text { nente) (2010 - 2011) } & \text { 5) Ações de implantação de ações e serviços de saúde (Implantação do Centro de } \\
& \text { Especialidade Odontológica - CEO) (2011-2017) } \\
\text { 6) Piso de Atenção Básica Variável (Aquisição de equipamentos odontológicos) } & \text { (2012-2013, 2016-2017) } \\
\text { 7) Implantação de ações e serviços de saúde (Incentivo adicional ao CEO) (2004- } \\
\text { 2009) }\end{array}$ \\
\hline
\end{tabular}

Fonte: Fundo Nacional de Saúde, 2018.

Já o bloco de financiamento de custeio da Média e Alta Complexidade é composto por a) Centro de Especialidades Odontológicas (CEO) municipal; b) CEO estadual e c) Fundos de Ações Estratégicas e Compensações (Faec) de vários tipos (quadro 1).

O bloco de investimento (capital) apresenta as rubricas específicas de ações em saúde bucal nos componentes 'Atenção Básica em Saúde bucal' (Aquisição de equipamentos e material permanente) (2010-2011); 'Ações de implantação de ações e serviços de saúde' (Implantação do Centro de Especialidade Odontológica CEO) (2011-2017) e 'Piso de Atenção Básica Variável' (Aquisição de equipamentos odontológicos) (2012-2013, 2016-2017).

No período de 2004 a 2009, o investimento relativo à implantação dos CEO constava no bloco Gestão do SUS, na rubrica de 'Implantação de ações e serviços de saúde' (Incentivo adicional ao CEO). Assim, independentemente do bloco em que se encontravam, consideraram-se os recursos de capital como investimento para implantação dos serviços.

Para a analisar o que representou o repasse da saúde bucal no montante total repassado pelo Ministério da Saúde, consideraram-se os gastos nos blocos AB, Média e Alta Complexidade, Assistência Farmacêutica, Gestão do SUS, Vigilância em Saúde, Investimentos e Diversos no período em estudo.

Os valores foram corrigidos pelo Índice Nacional de Preços ao Consumidor Amplo (IPCA), medido pelo Instituto Brasileiro de Geografia e Estatística (IBGE), para o mês de dezembro de 2017, a fim de que todos os valores pudessem ser alvo de comparação entre si. $\mathrm{O}$ IPCA é considerado como o índice que mede a inflação oficial no País. A correção dos valores foi efetuada pela calculadora oficial do Banco Central do Brasil, disponível no site: https:// www3.bcb.gov.br/CALCIDADAO.

A correção de valores pelo IPCA já vem sendo utilizada nos estudos em saúde, a exemplo do estudo ${ }^{18}$ que analisou os gastos com cigarro nas famílias brasileiras e do trabalho ${ }^{19}$ sobre o impacto do novo regime fiscal na saúde e na educação.

\section{Resultados e discussão}

A análise da série histórica dos repasses para saúde bucal no sistema público de saúde no período de 2003 a 2017 mostrou um gasto crescente até 2012, com queda no início de 2013, manutenção de valores de 2013 a 2016 e discreta redução em 2017 (tabela 1, gráfico 1). Os recursos destinados à $\mathrm{AB}$ representaram o maior montante dos repasses em todo o período estudado, o que diverge do valor global de repasse pelo FNS, que apresenta maior gasto com Média e Alta Complexidade. $\mathrm{Na}$ análise da participação percentual da $\mathrm{AB}$, Atenção Especializada (AE) e investimentos, observou-se uma redução do componente de $\mathrm{AB}$, aumento do de $\mathrm{AE}$ e queda nos investimentos (tabela 2, gráfico 1). Diferentemente dos repasses com a $A B$ em com a Saúde Básica que vêm apresentando redução, a Média Complexidade vem apresentando aumento, com certa manutenção 
nos valores a partir de 2014. Os investimentos representaram o menor gasto com saúde bucal quando comparado aos outros blocos.

Tabela 1. Forma de repasses federal fundo a fundo de financiamento de custeio e investimento por rubrica referente à saúde bucal, 2003-2017*

\begin{tabular}{lrrrr}
\hline Ano & $\begin{array}{r}\text { Repasses Atenção } \\
\text { Básica }\end{array}$ & $\begin{array}{r}\text { Repasse Atenção } \\
\text { Especializada }\end{array}$ & Investimento & Total de repasses \\
\hline 2003 & $\mathrm{R} \$ 180.711 .384,39$ & $\mathrm{R} \$ 4.199 .199,41$ & 0 & $\mathrm{R} \$ 184.910 .583,80$ \\
2004 & $\mathrm{R} \$ 400.253 .626,42$ & $\mathrm{R} \$ 13.190 .710,44$ & $\mathrm{R} \$ 6.304 .645,54$ & $\mathrm{R} \$ 419.748 .982,40$ \\
2005 & $\mathrm{R} \$ 546.418 .744,21$ & $\mathrm{R} \$ 45.331 .462,08$ & $\mathrm{R} \$ 31.877 .452,15$ & $\mathrm{R} \$ 623.627 .658,44$ \\
2006 & $\mathrm{R} \$ 688.235 .433,13$ & $\mathrm{R} \$ 92.765 .111,83$ & $\mathrm{R} \$ 24.652 .705,41$ & $\mathrm{R} \$ 805.653 .250,37$ \\
2007 & $\mathrm{R} \$ 782.892 .493,59$ & $\mathrm{R} \$ 120.313 .603,62$ & $\mathrm{R} \$ 8.195 .508,96$ & $\mathrm{R} \$ 911.401 .606,17$ \\
2008 & $\mathrm{R} \$ 804.365 .480,27$ & $\mathrm{R} \$ 120.555 .494,77$ & $\mathrm{R} \$ 7.993 .136,98$ & $\mathrm{R} \$ 932.914 .112,02$ \\
2009 & $\mathrm{R} \$ 831.562 .396,24$ & $\mathrm{R} \$ 139.337 .767,70$ & $\mathrm{R} \$ 6.868 .294,44$ & $\mathrm{R} \$ 977.768 .458,38$ \\
2010 & $\mathrm{R} \$ 919.183 .022,98$ & $\mathrm{R} \$ 125.605 .721,28$ & $\mathrm{R} \$ 444.078,79$ & $\mathrm{R} \$ 1.045 .232 .823,05$ \\
2011 & $\mathrm{R} \$ 938.858 .309,03$ & $\mathrm{R} \$ 149.397 .321,92$ & $\mathrm{R} \$ 10.661 .188,67$ & $\mathrm{R} \$ 1.098 .916 .819,62$ \\
2012 & $\mathrm{R} \$ 1.071 .567 .005,31$ & $\mathrm{R} \$ 166.756 .711,15$ & $\mathrm{R} \$ 7.584 .628,10$ & $\mathrm{R} \$ 1.245 .908 .344,56$ \\
2013 & $\mathrm{R} \$ 881.541 .675,52$ & $\mathrm{R} \$ 190.569 .481,50$ & $\mathrm{R} \$ 10.302 .606,98$ & $\mathrm{R} \$ 1.082 .413 .764,00$ \\
2014 & $\mathrm{R} \$ 880.880 .671,40$ & $\mathrm{R} \$ 233.202 .804,46$ & $\mathrm{R} \$ 3.881 .009,69$ & $\mathrm{R} \$ 1.117 .964 .485,55$ \\
2015 & $\mathrm{R} \$ 887.097 .152,31$ & $\mathrm{R} \$ 215.160 .162,46$ & $\mathrm{R} \$ 1.739 .921,08$ & $\mathrm{R} \$ 1.103 .997 .235,85$ \\
2016 & $\mathrm{R} \$ 909.189 .917,30$ & $\mathrm{R} \$ 217.513 .800,79$ & $\mathrm{R} \$ 2.651 .231,75$ & $\mathrm{R} \$ 1.129 .354 .949,84$ \\
2017 & $\mathrm{R} \$ 829.995 .247,00$ & $\mathrm{R} \$ 226.725 .301,48$ & $\mathrm{R} \$ 2.835 .000,00$ & $\mathrm{R} \$ 1.059 .555 .548,48$ \\
\hline
\end{tabular}

Fonte: Fundo Nacional de Saúde, Brasil, 2017

*Valores corrigidos pelo IPCA - IBGE em dezembro de 2017.

Gráfico 1. Financiamento federal da PNSB por bloco de financiamento (custeio e investimento) entre 2003 e 2017

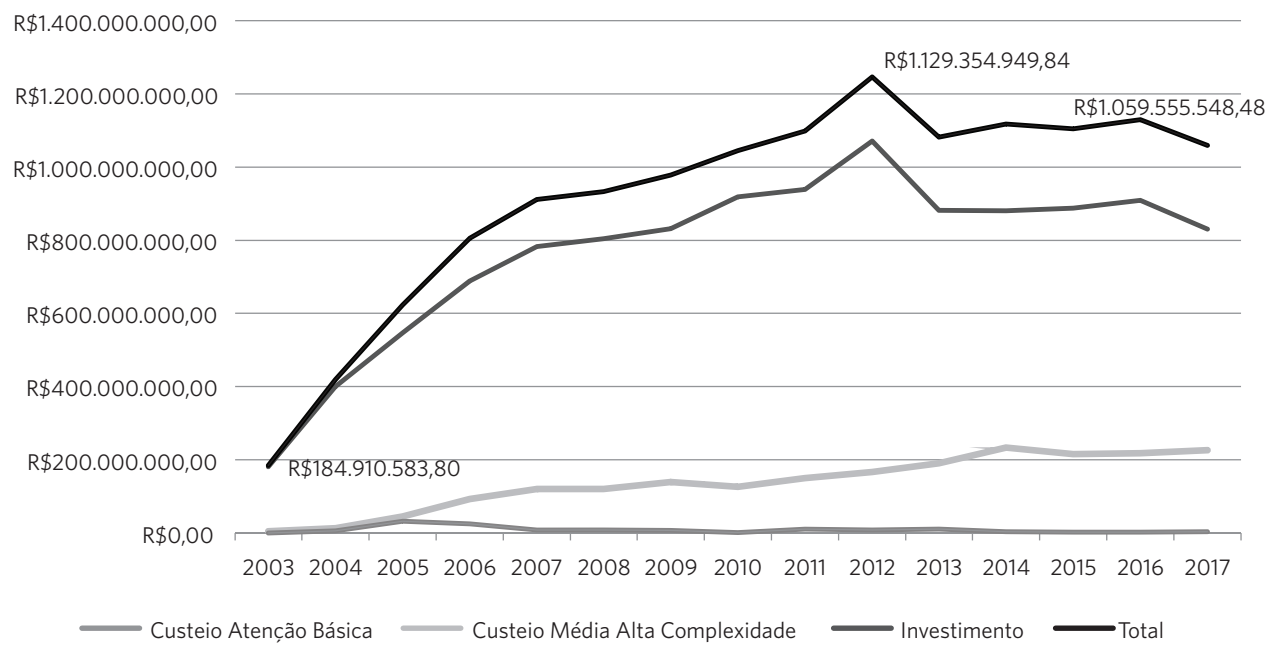


Pode-se afirmar que a primeira e segunda gestão do governo Lula (2003-2010) foram caracterizadas como período de maior crescimento nos repasses relacionados com a saúde bucal. Esse dado corrobora outro trabalho que analisou o governo Lula, tendo apontado que a implementação da PNSB entrou na janela de oportunidade da agenda governamental e fez parte do cumprimento das promessas de campanha, o que conferiu destaque de forma crescente ${ }^{20}$.

O governo federal ainda é o maior detentor de recursos e principal agente responsável da política de saúde em todo o território nacional5.
O grande aumento no repasse de 2003 a 2006 é justificado pela crescente implantação de ESB no Programa de Saúde da Família e de CEO. Esse período de maior crescimento e expansão das equipes de $\mathrm{AB}$ e $\mathrm{AE}$ no primeiro governo Lula (tabela 2) correspondeu à implantação inicial das ações da política Brasil Sorridente. Observa-se que os gastos em saúde bucal representavam $0,62 \%$ em 2003, passando para $1,75 \%$ em 2007, final do governo Lula I (gráfico 1). Destaca-se que a velocidade de expansão das ESB foi menor nos outros governos.

Tabela 2. Número de ESB, CEO implantados, total de repasse do governo federal e porcentagem de gasto na $A B, A E$ e investimento entre 2003 e 2017

\begin{tabular}{lrrrrrr}
\hline \multirow{2}{*}{ Ano } & \multicolumn{2}{c}{ Oferta de serviços } & \multicolumn{5}{c}{ Financiamento Federal } \\
\cline { 2 - 7 } & ESB (mod. I e II) & CEO & Total de repasses (R\$) & Repasses AB & Repasse AE & Investimento \\
\hline 2003 & 617 & - & $R \$ 184.910 .583,80$ & $97,7 \%$ & $2,3 \%$ & $0,0 \%$ \\
2004 & 8.951 & 100 & $R \$ 419.748 .982,40$ & $95,4 \%$ & $3,1 \%$ & $1,5 \%$ \\
2005 & & & $R \$ 623.627 .658,44$ & $87,6 \%$ & $7,3 \%$ & $5,1 \%$ \\
2006 & 15.086 & 498 & $R \$ 805.653 .250,37$ & $85,4 \%$ & $11,5 \%$ & $3,1 \%$ \\
2007 & 15.694 & 604 & $R \$ 911.401 .606,17$ & $85,9 \%$ & $13,2 \%$ & $0,9 \%$ \\
2008 & 17.801 & 674 & $R \$ 932.914 .112,02$ & $86,2 \%$ & $12,9 \%$ & $0,9 \%$ \\
2009 & 18.982 & 808 & $R \$ 977.768 .458,38$ & $85,0 \%$ & $14,3 \%$ & $0,7 \%$ \\
2010 & 20.424 & 853 & $R \$ 1.045 .232 .823,05$ & $87,9 \%$ & $12,0 \%$ & $0,0 \%$ \\
2011 & 21.425 & 882 & $R \$ 1.098 .916 .819,62$ & $85,4 \%$ & $13,6 \%$ & $1,0 \%$ \\
2012 & 22.203 & 944 & $R \$ 1.245 .908 .344,56$ & $86,0 \%$ & $13,4 \%$ & $0,6 \%$ \\
2013 & 23.150 & 988 & $R \$ 1.082 .413 .764,00$ & $81,4 \%$ & $17,6 \%$ & $1,0 \%$ \\
2014 & 24.279 & 1.030 & $R \$ 1.117 .964 .485,55$ & $78,8 \%$ & $20,9 \%$ & $0,3 \%$ \\
2015 & 24.467 & 1.034 & $R \$ 1.103 .997 .235,85$ & $80,4 \%$ & $19,5 \%$ & $0,2 \%$ \\
2016 & 24.384 & 1.072 & $R \$ 1.129 .354 .949,84$ & $80,5 \%$ & $19,3 \%$ & $0,2 \%$ \\
2017 & 25.905 & 1.115 & $R \$ 1.059 .555 .548,48$ & $78,3 \%$ & $21,4 \%$ & $0,3 \%$ \\
\hline
\end{tabular}

Fonte: Elaboração própria baseada em dados da Sala de apoio à Gestão Estratégica (Sage)/Departamento de Atenção Básica (DAB)/ Secretaria de Atenção à Saúde do Ministério da Saúde (SAS/MS).

Nota: Referência dezembro de cada ano. 
Estudos sobre as ESB em municípios brasileiros apontaram a indução federal, a partir do ano 2000, como grande protagonista na implantação e desenvolvimento das ESB21,22. Há gestores municipais que justificam não ter implantado a saúde bucal na $\mathrm{AB}$ antes do referido ano, quando foi publicada a Portaria de $\mathrm{n}^{0} 144$ do Ministério da Saúde de incentivo financeiro à ESB na Estratégia Saúde da Família (ESF), diante dos altos custos dos equipamentos e materiais odontológicos ${ }^{21}$.

Existe uma grande desigualdade entre os municípios no que tange à capacidade de captação de recursos, execução das políticas de saúde e manutenção dos serviços, sobretudo naqueles com piores indicadores de saúde e os localizados no interior dos estados ${ }^{21}$. Esse estudo $^{21}$ verificou que há maior número de ESB tipo II, ou seja, com cirurgião-dentista, auxiliar em saúde bucal e técnico em saúde bucal, em municípios com Índice de Desenvolvimento Humano (IDH) muito alto, na região Sudeste, com cobertura menor que $50 \%$ e proporção de exodontias menor que $8 \%$. Além disso, estudos de análise do financiamento federal a partir de uma perspectiva regional revelaram os efeitos da indução federal na direção da oferta de serviços em saúde bucal com maior equidade. Outro trabalho ${ }^{23}$ destacou a expansão das ESB até 2007, atribuindo tal fato também à implantação do Brasil Sorridente e à garantia do financiamento dentro de um sistema com muitas outras prioridades.

Em 2011, a participação federal nos gastos públicos em saúde foi de apenas $45,5 \%$, inferiores ao financiamento de estados e municípios juntos. No primeiro governo de Dilma Rousseff (2011-2014), observou-se um incremento inicial e posterior queda no financiamento na saúde bucal, patamar que se manteve no início do seu segundo governo em $2015^{5}$. No ano de 2016, observou-se novo decréscimo relativo aos repasses em estudo no governo de Michel Temer. A diferença nestes dois últimos anos refere-se ao decréscimo no custeio da $\mathrm{AB}$, apesar do número de ESB ter aumentado discretamente em 2016. O que pode explicar essa diferença é a discrepância de valores repassados mês a mês com o descredenciamento mensal de equipes e recredenciamento de outras em meses subsequentes.

Quanto aos investimentos, observaram-se picos com aumento de repasse nos anos de 2011 e 2013, o que pode ter correspondido aos recursos para implantação de novos CEO e aquisição de novos equipamentos.

Entre os anos de 2012 e 2013, observou-se queda no financiamento da $\mathrm{AB}$, o que não pode ser explicado pela redução na implantação de novas ESB, pois houve aumento de 947 equipes. Quanto à $\mathrm{AE}$, o único ano em que houve uma discreta redução no repasse financeiro foi 2010, o que também não pode ser justificado pelo número de CEO implantados, tendo em vista que houve aumento de 45 serviços especializados.

A análise percentual por componente, comparando os anos de 2015, 2016 e 2017, apontou que houve manutenção percentual do custeio da saúde bucal na AB em 2015 (79,5\%) e 2016 (79,7\%), com diminuição em 2017.

Em 2016, é possível explicar parte do aumento do montante de recursos pela variação mensal das ESB implantadas nesse ano e pelo repasse de $20 \%$ a $60 \%$ no custeio às equipes que foram avaliadas pelo Programa de Melhoria do Acesso e da Qualidade dos Centros de Especialidades Odontológica (PMAQ-CEO). No ano de 2017, o componente CEO explica parte do incentivo do PMAQCEO pelo incentivo às unidades que aderiram à rede da pessoa com deficiência.

Ainda em relação ao montante global de recursos repassados pelo FNS/Ministério da Saúde, observou-se um aumento no período inicial, manutenção de 2006 a 2012, queda em 2013 e outro decréscimo em 2016 (gráfico 2). Pesquisadores apontaram que a política de saúde bucal recebeu prioridade no Ministério da Saúde pelo recurso repassado e pela expansão das equipes ${ }^{24}$. Em 2015 e 2014, no bloco de investimento (capital), verificou-se apenas o repasse de recursos no componente relativo ao CEO. 
Gráfico 2. Composição percentual do financiamento da atenção à saúde bucal (custeio e capital) em relação aos repasses realizados pelo FNS, 2003 e 2017

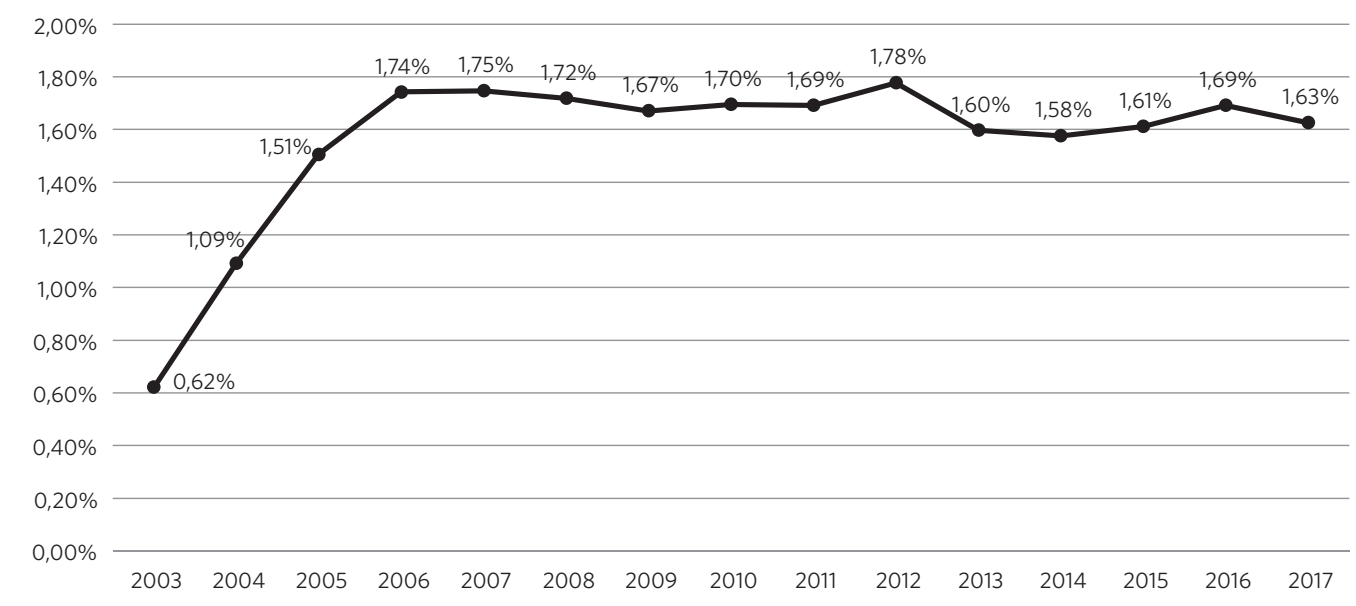

Fonte: Fundo Nacional de Saúde, 2018.

Ainda para o ano de 2016, quanto à AE, a maior diferença de repasses esteve relacionada com os CEO municipais. A seguir, observaram-se os maiores gastos com a Alta Complexidade (Faec-Sistema de Informação Ambulatorial - SIA - Tratamentos Odontológicos/Faec-SIA - Órteses/Próteses/ Materiais - OPM em Odontologia). O maior financiamento em 2016 não se revelou na maior oferta de serviços públicos odontológicos. A ampliação da implantação das ESB na $\mathrm{AB}$ não ocorreu e houve redução de CEO e Laboratórios Regionais de Prótese.

Houve discreta ampliação na implantação das ESB na AB em 2017. Na série histórica analisada, o único ano que encerrou com redução do número de ESB na ESF foi o ano de 2016.

Em 2017, evidenciou-se um aumento da participação percentual do financiamento da AE, especialmente a partir de 2013. O financiamento da AE era de apenas 2,3\% em 2003, chegando a $17,6 \%$ em 2013, 20,9\% em 2014, $19,3 \%$ em 2016 e $21,4 \%$ em 2017. Portanto, o recurso financeiro repassado refletiu a prioridade dessa política de expansão da atenção odontológica especializada no SUS (tabela 2). Estudo apontou o avanço na expansão dos
CEO, ratificando o papel central que a $\mathrm{AE}$ vem ganhando nessa política, tendo em vista ser a primeira ação federal mais abrangente para a AE em saúde bucal ${ }^{25}$.

Entre os centros especializados, os montantes maiores foram para os municipais, e os CEO estaduais representaram pequena parte (1,4\%). Já o custeio de procedimentos de Alta Complexidade, por meio do Faec, conforme já referido, foi de $2,68 \%$, entre repasses a estados e municípios, assim como aos próprios prestadores federais (quadro 1).

Os repasses financeiros do Ministério da Saúde no período de 1998 a 2016 relacionados com a saúde bucal receberam em média $1,40 \%$ dos valores repassados para a saúde ${ }^{5}$. Coadunando com os achados do presente estudo, é possível verificar, nesse período, o crescimento real nos repasses, com estabilização nos últimos anos e tendência à queda, além da perda de espaço da saúde bucal dentro dos blocos de financiamento para outras ações e programas do governo federal ${ }^{5}$.

Embora a proposta do estudo tenha como base o monitoramento dos repasses federais da PNSB no período de 2003 a 2017, é importante destacar que, de acordo com a descentralização 
da gestão e desconcentração de recursos, municípios e estados contribuem com valores próprios na atenção à saúde bucal7,8.

Após o cumprimento dos ritos legislativos da PEC 241 na Câmara dos Deputados e renomeada em PEC 55, para tramitação no Senado Federal, as medidas de austeridade fiscal foram aprovadas em 2016, com a determinação de um teto financeiro máximo para a saúde até 2036. $\mathrm{O}$ valor ficou restrito ao gasto do ano anterior somado com o reajuste da inflação, cujos repasses não acompanharão o crescimento populacional e da renda. Outro possível impacto será o fim dos blocos de financiamento como até então eram repassados os recursos, que dificultará tanto a prioridade da saúde bucal quanto o seu acompanhamento. O impacto na PNSB aponta para um cenário de não obrigatoriedade na manutenção do financiamento para essa política específica, com diminuição dos repasses de custeio, instabilidade nos investimentos de capital, o que pode ocasionar sucateamento da rede de cuidados ${ }^{19}$.

Em conclusão, cabe destacar que houve redução discreta do financiamento federal no ano de 2017 quando comparado a 2016. Contudo, ainda que tenha havido redução, chama a atenção uma certa inconsistência entre a implantação da política, seus resultados e o financiamento já apontado em estudo anterior ${ }^{26}$. Observou-se um aumento da participação percentual do financiamento da $\mathrm{AE}$ na saúde, especialmente a partir de 2013, e redução na participação percentual relativa à $\mathrm{AB}$ e aos investimentos. Obviamente, é importante ressaltar que o repasse federal não significa necessariamente que esse recurso foi efetivamente gasto pelo município ou estado, ou seja, analisar a relação entre o orçado e efetivamente gasto mereceria estudos distintos.

Estudos sobre sistemas de informação, análises de implantação e resultados da política nos âmbitos regional e municipal são necessários para elucidar os diversos fatores relacionados com os achados deste estudo. Manteve-se uma tendência de ampliação do financiamento da AE. Contudo, sem uma atenção primária fortalecida, esse esforço pode não produzir o resultado da integralidade pretendida. Destaca-se que os repasses municipais e estaduais não foram objeto de estudo do presente trabalho, merecendo estudos posteriores.

O modelo de financiamento proposto pela PEC 55 legitima medidas de austeridade fiscal, indicando um caminho institucionalizado de redução dos repasses federais na saúde, com consequente depreciação do SUS e efeitos negativos na saúde bucal, que necessitam ser analisados em estudos posteriores.

\section{Colaboradores}

TRA Rossi participou da formulação da pesquisa, coleta e análise dos dados, escrita e revisão final do manuscrito. SCL Chaves coordenou a pesquisa, participou da análise dos dados e revisou o manuscrito final. AMFL Almeida, CML Santos e SF Santana participaram da análise dos dados, escrita do artigo e revisão final. 


\section{Referências}

1. Moimaz SAS, Garbin CAS, Garbin AJI, et al. Desafios e dificuldades do financiamento em saúde bucal: uma análise qualitativa. Rev. de Adm. Pública [internet]. 2008 [acesso em 2018 jul 1]; 42(6):1121-1135. Disponível em: http://hdl.handle.net/11449/15416.

2. Piola SF, Servo ML, Sá EB, et al. Financiamento do Sistema Único de Saúde: Trajetória recente e cenários para o futuro. Análise econômica. 2012 set; 30(esp):9-33.

3. Franco DH. Financiamento público e privado da saúde bucal no Brasil e no mundo. [tese]. [São Paulo]: Universidade Estadual de Campinas; 2017. 258 p.

4. Teixeira HV, Teixeira MG. Financiamento da saúde pública no Brasil: a experiência do Siops. Ciênc. Saúde Colet. [internet]. 2003 [acesso em 2018 jun 4]; 8(2):379-391. Disponível em: http://www.scielo.br/ pdf/csc/v8n2/a05v08n2.pdf.

5. Mendes A, Marques RM. O financiamento da Atenção Básica e da Estratégia Saúde da Família no Sistema Único de Saúde. Saúde debate [internet]. 2014 [acesso em 2018 jul 12]; 38(103):900-916. Disponível em: https://doi.org/10.5935/0103-1104.20140079.

6. Soares A, Santos NR. Financiamento do Sistema Único de Saúde nos governos FHC, Lula e Dilma. Saúde debate [internet]. 2014 mar [acesso em 2018 jul. 01]; 38(100):18-25. Disponível em: http://www. scielo.br/scielo.php?script=sci_arttext\&pid=S0103$-11042014000100018 \& \operatorname{lng}=$ en\&nrm=iso.

7. Macêdo MSR, Chaves SCL, Fernandes ALC. Investimentos e custos da atenção à saúde bucal na Saúde da Família. Rev. Saúde Púb. [internet]. 2016 [acesso em 2018 jun. 14]; 50(41):1-12. Disponível em: http:// www.redalyc.org/pdf/672/67247719048.pdf.

8. Roa AC. Financiamento dos sistemas de saúde na América do Sul. In: Sistema de saúde no Brasil: organização e financiam, organizadores. Rio de Janeiro: ABrES; Ministério da Saúde; OPAS/OMS; 2016. p. $75-100$.
9. Castro ALB, Machado CV. A política de atenção primária à saúde no Brasil: notas sobre a regulação e o financiamento federal. Cad. Saúde Pública. 2010 abr. 26(4):693-705.

10. Melamed C, Costa NR. Inovações no financiamento federal à Atenção Básica. Ciênc. Saúde Colet. [internet]. 2003 [acesso em 2018 jun 27]; 8(2):393-401. Disponível em: http://dx.doi.org/10.1590/S141381232003000200006 .

11. Porto SM, Uga MAD, Moreira RS. Uma análise da utilização de serviços de saúde por sistema de financiamento: Brasil 1998-2008. Ciênc. Saúde Colet. [internet]. 2011 [acesso em 2018 jun 27]; 16(9):37953806. Disponível em: http://www.scielo.br/scielo. php?script=sci_arttext\&pid=S141381232011001000 $015 \& \operatorname{lng}=$ en $\& n r m=$ iso.

12. Santos IS, Uga MAD, Porto SM. O mix público-privado no Sistema de Saúde Brasileiro: financiamento, oferta e utilização de serviços de saúde. Ciênc. Saúde Colet. [internet]. 2008 [acesso em 2018 jun. 27]; 13(5):1431-1440. Disponível em: http://www.scielo. br/pdf/csc/v13n5/09.pdf.

13. Vazquez DA. Efeitos da regulação federal sobre o financiamento da saúde. Cad. Saúde Pública [internet]. 2011 [acesso em 2018 jun 27]; 27(6):1201-1212. Disponível em: http://www.scielo.br/pdf/csp/v27n6/17.pdf.

14. Sobrinho JEL, Santo ACGE. Análise dos custos da saúde bucal na atenção básica no município de Vitória de Santo Antão. Arq. Odontol. Belo Horizonte. 2013 mar; 49(1):26-31.

15. Fernandes MM, Daruge-Junior E, Oliveira MR, et al. Relato de investigação relacionada ao financiamento de ortodontia pela rede pública. Odonto São Bernardo do Campo. 2011; 19(37):49-53.

16. Kornis GEM, Maia LS, Fortuna RFP. Evolução do financiamento da atenção à saúde bucal no SUS: uma análise do processo de reorganização assistencial 
frente aos incentivos federais. Physis. Rio de Janeiro 2011; 21(1):197-215.

17. Nóbrega CBC, Hoffmann RHS, Pereira AC, et al. Financiamento do setor saúde: uma retrospectiva recente com uma abordagem para a odontologia. Ciênc. Saúde Colet. [internet]. 2010 jun [acesso em 2018 jul 13]; 15(supl1):1763-1772. Disponível em: http://www.scielo.br/scielo.php?script=sci_ arttext\&pid=S1413-81232010000700088\&lng=en . http://dx.doi.org/10.1590/S1413-81232010000700088.

18. Garcia LP, Sant'Anna AC, Freitas LRS, et al. A política antitabagismo e a variação dos gastos das famílias brasileiras com cigarro: resultados das Pesquisas de Orçamentos Familiares, 2002/2003 e 2008/2009. Cad. Saúde Pública [internet]. 2015 set [acesso em 2018 jun 27]; 31(9):1894-1906. Disponível em: http://www.scielo.br/pdf/csp/v31n9/0102-311X-csp-31-9-1894.pdf.

19. Rossi P, Dweck E. Impactos do novo regime fiscal na saúde e educação. Cad. Saúde Pública [internet]. 2016 dez [acesso em 2018 jun 27]; 32(12):1-5. Disponível em: http://www.scielo.br/pdf/csp/v32n12/16784464-csp-32-12-e00194316.pdf.

20. Menicucci TM. A política de saúde no governo Lula. Saúde soc. [internet]. 2011 jun [acesso 2018 jun 28]; 20(2):522-532. Disponível em: http://www.scielo.br/ pdf/sausoc/v20n2/22.pdf.

21. Mattos GCM, Ferreira EF, Leite ICG, et al. A inclusão da equipe de saúde bucal na Estratégia Saúde da Família: entraves, avanços e desafios. Ciênc. Saúde Colet. [internet]. $2014 \mathrm{fev}$ [acesso em 2018 jul 13]; 19(2):373-382. Disponível em: http://www.scielo.br/ pdf/csc/v19n2/1413-8123-csc-19-02-00373.pdf.
22. Almeida-Junior LJS, Vieira DRP, Rodrigues VP, et al. A inserção da saúde bucal na Estratégia de Saúde da Família no estado do Maranhão (2002-2011). Rev Pesq Saúde. 2014 set-dez; 15(3):356-359.

23. Martins PHS, Amaral-Junior OL, Faustino-Silva DD, et al. Desigualdades na distribuição das equipes de saúde bucal no Brasil. Stomatos [internet]. 2017 jul-dez [acesso em 2018 jun 29]; 23(45):4-13. Disponível em: http://www.periodicos.ulbra.br/index.php/ stomatos/article/viewFile/3130/2830.

24. Teixeira CF, Paim JS. A política de saúde no governo Lula e a dialética do menos pior. Saúde debate [internet]. 2005 [acesso em 2018 jun 29]; 29(71):268283. Disponível em: http://www.redalyc.org/ pdf/4063/406345256005.pdf.

25. Pedrazzi V, Dias KRHC, Rode SM. Oral health in Brazil - Part II: Dental Specialty Centers (CEOs). Braz. oral res. [internet]. 2008 ago [acesso em 2018 jun 29]; 22(supl):18-23. Disponível em: http://www.scielo.br/ pdf/bor/v22sl/04.pdf.

26. Chaves SCL, Almeida AMFL, Rossi TRA, et al. Política de Saúde Bucal no Brasil 2003-2014: cenário, propostas, ações e resultados. Ciênc. Saúde Colet. [internet]. 2017 jun [acesso em 2018 nov 7]; 22(6):1791-1803. Disponível em: http://www.scielo.br/scielo.php?script=sci_arttext $\&$ pid $=$ S1413$-81232017002601791 \& \operatorname{lng}=\mathrm{en}$.

Recebido em 15/07/2018

Aprovado em 07/10/2018

Conflito de interesses: inexistente

Suporte financeiro: não houve 